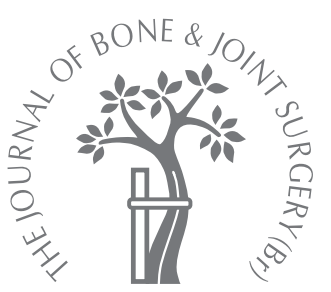

\title{
Precision tumour resection and reconstruction using image-guided computer navigation
}

\author{
K. C. Wong, \\ S. M. Kumta, \\ K. H. Chiu, \\ G. E. Antonio, \\ P. Unwin, \\ K. S. Leung
}

From The Prince of

Wales Hospital,

Hong Kong, China

\begin{abstract}
The use of a navigation system in musculoskeletal tumour surgery enables the integration of pre-operative CT and MRI images to generate a precise three-dimensional anatomical model of the site and the extent of the tumour.

We carried out six consecutive resections of musculoskeletal tumour in five patients using an existing commercial computer navigation system. There were three women and two men with a mean age of 41 years (24 to 47 ). Reconstruction was performed using a tumour prosthesis in three lesions and a vascularised fibular graft in one. No reconstruction was needed in two cases. The mean follow-up was 6.9 months (3.5 to 10). The mean duration of surgery was 28 minutes (13 to 50 ). Examination of the resected specimens showed clear margins in all the tumour lesions and a resection that was exactly as planned.
\end{abstract}

The use of a navigation system allows precise resection of a tumour and accurate reconstruction of the resultant defect thereby sparing important anatomical structures and preserving function, particularly in anatomically awkward areas.

Wide-local resection with an adequate margin is a crucial step in the management of patients with a musculoskeletal malignancy. If a custom-made prosthesis is to be used for reconstruction, the plane of resection must not only be at the correct level, but should also be appropriately orientated in order to accommodate the prosthesis and restore satisfactory function. This is particularly relevant in difficult areas such as the pelvis, or when an intercalary joint-sparing resection is contemplated.

Surgical planning for a wide resection and endoprosthetic replacement requires detailed analysis of the pre-operative imaging. The tumour surgeon (KCW, SMK) plans for a resection with clear margins along a desired plane using identifiable anatomical landmarks and measurements based on radiographs. However, without the benefit of a precise intraoperative guidance system, discrepancies between the planned resection and that achieved may be expected, particularly in cases of pelvic or diaphyseal resection. Computeraided navigation has improved precision in spinal surgery, trauma, and joint replacement. ${ }^{1-5}$ It involves the generation of a virtual image which allows the surgeon to receive real-time visual feedback on the precise location of sur- gical instruments and guides. It can be manipulated to allow the surgeon to view the patient's anatomy in any desired orientation, thereby enabling him to site implants with greater accuracy.

We have adapted an existing navigation system for use in musculoskeletal tumour resection by integrating MR images (Stryker Navigation, CT spine Version 1.60, Stryker Pacific Ltd, Hong Kong, China) with CT-based anatomical data. This enables us to model not only the relevant anatomy, but also the intraand extra-osseous extent of the tumour. In this paper we report our experience of using this system in five patients with six tumours of the pelvis or extremities.

\section{Patients and Methods}

Between January 2006 and September 2006, six surgical resections were performed in five patients with bone tumours with the assistance of the image-guided computer navigation system. The mean age of the patients was 41 years (24 to 47 ) (Table I). There were three pelvic metastases, one recurrent sacral chordoma, one sarcoma of the proximal femur and one parosteal osteosarcoma of the proximal tibia. Intra-operative guidance was thought to be necessary either because of anticipated difficulties in achieving an accurate tumour resection or in attaining a satisfactory resection plane to accommodate a tumour prosthesis.

Pre-operative planning. Pre-operative CT and MRI examinations of each patient were per- 
Table I. Details of six cases with tumour resection under the guidance of computer navigation

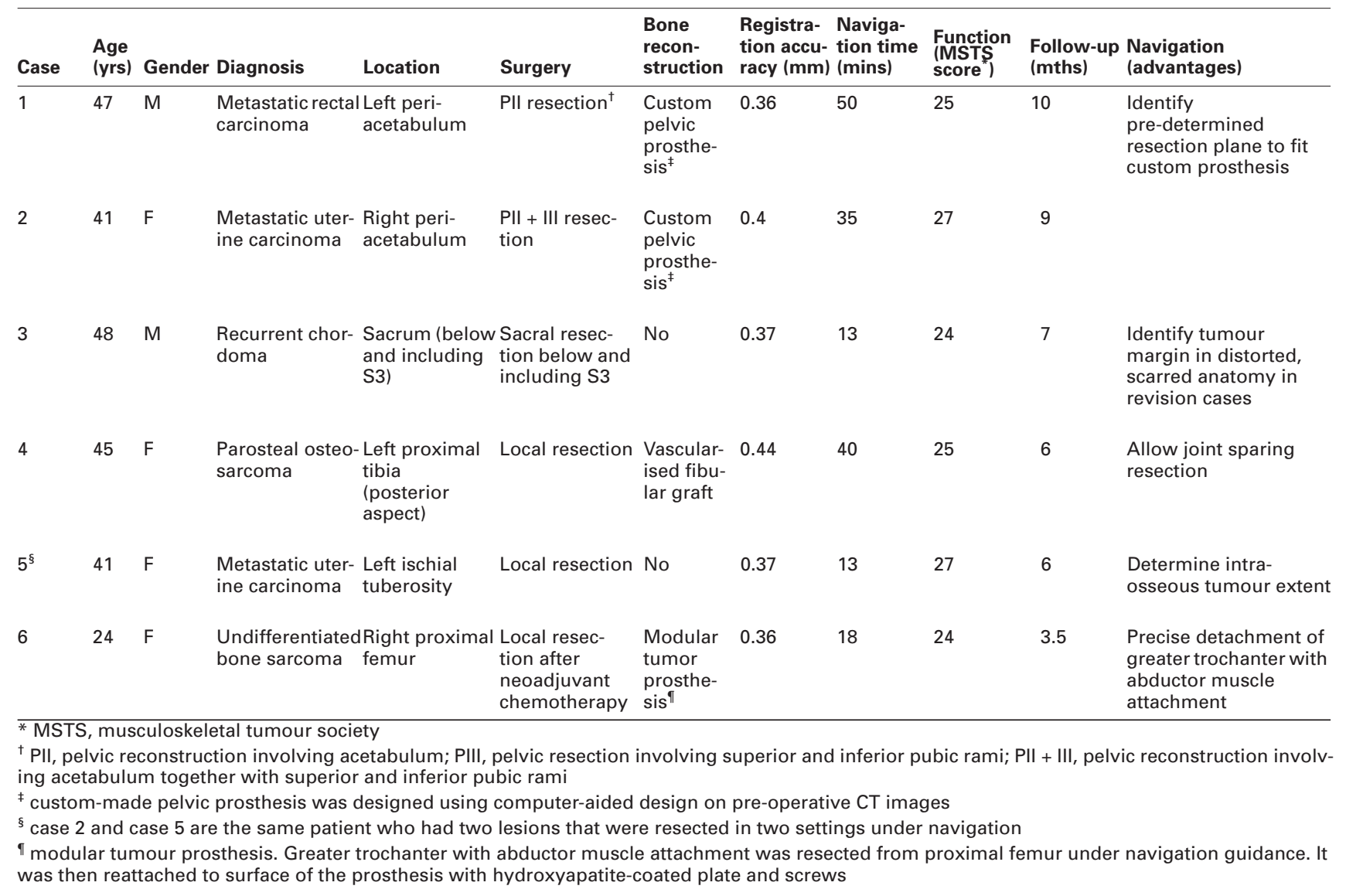

formed. Radiological data were obtained and reformatted into coronal, axial, sagittal and three-dimensional (3D) views of the bone using the navigation system. Because no specific software had been developed for the resection of bone tumour, we used that developed to assist in the placement of pedicle screws. The CT and MR images for three patients were fused using the navigation software and the tumour margin marked (Fig. 1). The MRI dimensions of the tumour were extracted onto a 3D image generated by the navigation system. These reconstructed images allowed pre-operative surgical planning. The plane of tumour resection was defined and marked using multiple virtual screws sited along the margin of the planned resection (Fig. 2). In cases 1 and 2, we also integrated computer-aided design (CAD) data on the custom-made pelvic prosthesis provided by the manufacturer (Stanmore Implants Worldwide Ltd, Middlesex, United Kingdom). In case 1, we used the patient's CT data to generate a plaster model of the pelvic bone with a rapid prototyping machine (ZPrinter 3ol, Z Corporation, Burlington, Massachussetts). The navigationassisted resection was successfully rehearsed and executed on the plaster model before the definitive operation.

Intra-operative navigation and surgical resection. Each surgical resection was performed using navigation. After the appropriate exposure, a tracker was attached to the bone in which the tumour was located. This enabled us to carry out the process of registration and match precisely the operative anatomy to the virtual image generated by the navigation software. Registration involved the matching of four selected bony landmarks, and at least 35 points were chosen on the exposed surface of the bone. The navigation software calculated the registration errors which indicated any mismatch between the computer-generated images and the patient's anatomy. Only if there was accurate realtime matching between the anatomy and the virtual images could we rely on the accuracy of the navigation system. Next, the navigation probe and those instruments mounted with navigation trackers (drill and diathermy) were calibrated to the system. This allowed the real-time spatial location of these instruments to be displayed in relation to the patient's anatomy on the $3 \mathrm{D}$ virtual image (Fig. 3).

We next located the anatomical position of the virtual screws on our patient, using navigated tools under guidance, and marked their position with diathermy. The diathermy marks were joined to indicate the resection plane, an oscillating saw was used to make the osteotomy and the tumour removed en bloc.

The skeletal defect was reconstructed using a custommade pelvic prosthesis in two patients, a modular prox- 


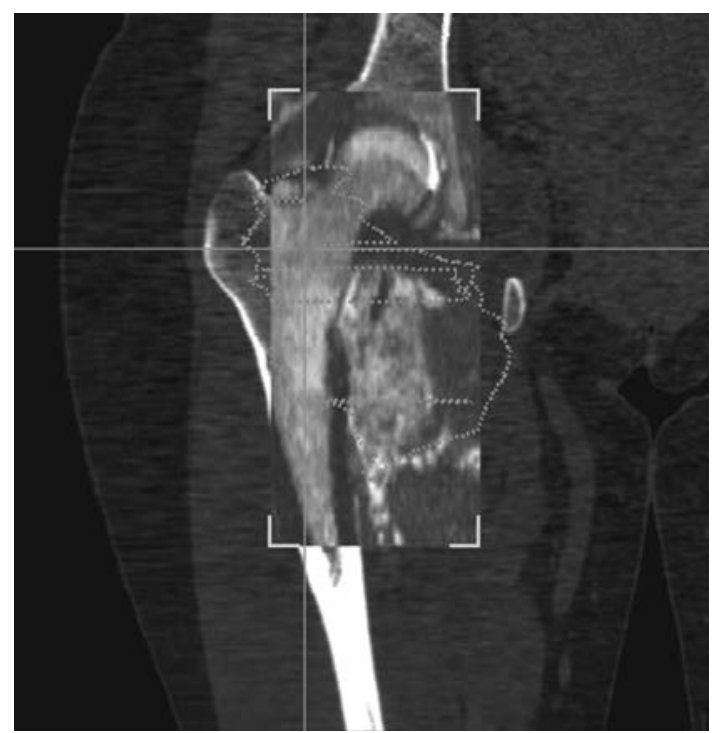

Fig. 1

$\mathrm{CT}$ and MR images were fused in the navigation system. The tumour was delineated from the MR images. A three-dimensional CT bone and tumour model was then generated for subsequent navigation planning and intra-operative execution.

imal femoral prosthesis in one, and a vascularised fibular graft in one. No reconstruction was required for two patients. Image intensification was not needed at any point in the whole procedure.

The data recorded included the time for pre-operative planning using the navigation system; the time for operative set-up and the execution of the navigation procedures; the registration error; the histological evaluation of the resected specimens for tumour margins and the function outcome using the Musculoskeletal Tumour Society (MSTS) score. ${ }^{6}$

\section{Results}

Although the pre-operative planning for navigation took a mean of three hours (one to six), the mean time for navigation procedures during surgery was 28 minutes (13 to 50). The navigation time lessened with practice. The mean registration error was $0.43 \mathrm{~mm}$ (0.35 to 0.63$)$. The virtual preoperative CT images correlated well with the patients' anatomy at registration. The two custom-made pelvic prostheses fitted exactly as planned (Fig. 4). Histological examination of all the resected specimens showed a clear tumour margin. In each case the resection achieved was exactly as planned.

The cross-section of pelvic bone after iliac osteotomy was identical to that seen on the computer-aided design (CAD) dataset of the custom-made pelvic prosthesis in case 1. The CT images of the resected specimen were obtained in the patient with a proximal femoral tumour (case 6), and were merged with the pre-operative CT images. The dimensions of the resected specimen, including the plane of resec-

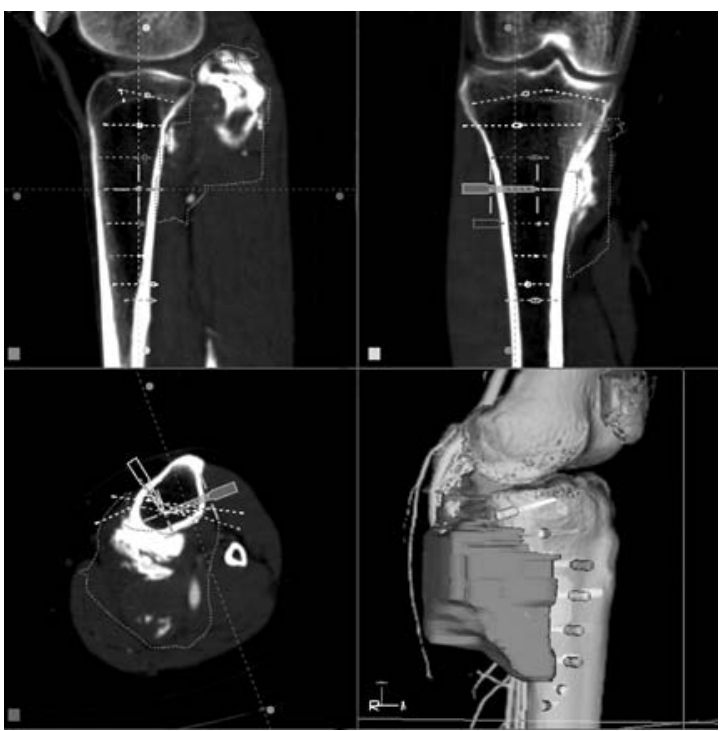

Fig. 2

For the three dimensional CT bone and tumour model, the plane of tumour resection was formed by putting multiple virtual screws along the intended resection in the pre-operative planning in case 4 .

tion, were exactly as planned. The mean functional MSTS score was 25.3 (24 to 27) after a mean follow-up of 6.9 months (3.5 to 10$)$.

\section{Discussion}

There is no commercially available navigation software package for surgery of a musculoskeletal tumour. This paper describes how we adapted a commercially available navigation system for surgery of the spine for our requirements. The real-time visualisation of skeletal anatomy proved particularly useful when carrying out resections of the pelvis and sacrum.

This instant visual feedback of 3D images allows the surgeon to obtain a precise sense of orientation, not only with reference to the relevant bony anatomy, but in relation to the intra-osseous extent of the tumour. Without navigation, this oncologically significant landmark can only be imagined and is impossible to locate on the bone surface. Safe margins can be obtained without navigation but may require the resection of additional bone, thereby jeopardising the fit of a prosthesis that has been precisely custom-made to fit a resected segment of bone. Similarly, the use of navigation enabled us to perform a joint-sparing tibial resection, which would not have been possible without an accurate guide to the plane of resection.

Navigation-assisted resection of bone has only been reported in two small case series. Hufner et $\mathrm{al}^{7}$ successfully used a navigated chisel to identify the extent of tumour in three patients with sacral tumours, and Krettek et $\mathrm{al}^{8}$ used computer-aided resection in two patients. Neither used preoperative planning, nor did they plan the plane of resection by fusing CT and MR images, but they did so intra- 

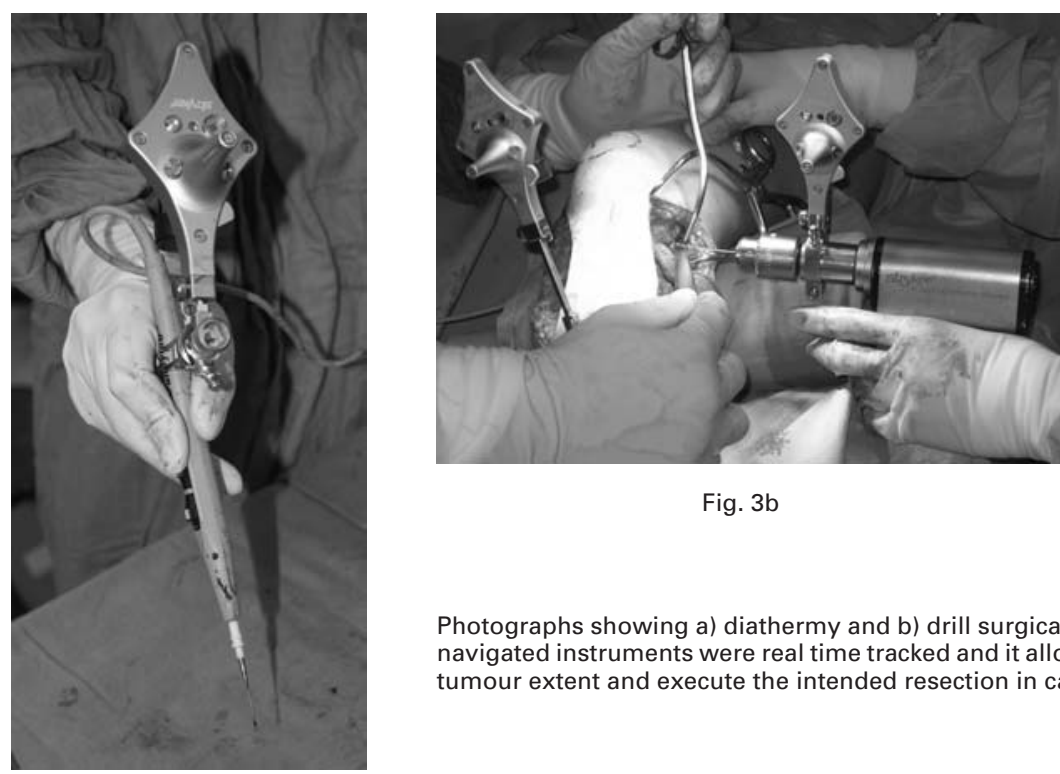

Fig. $3 b$ tumour extent and execute the intended resection in case 1.

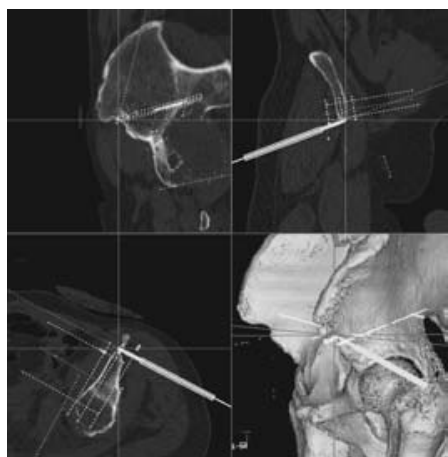

Fig. 3c

Photographs showing a) diathermy and b) drill surgical instruments with navigation tracker. c) The navigated instruments were real time tracked and it allowed surgeons to visualise the intra-osseous

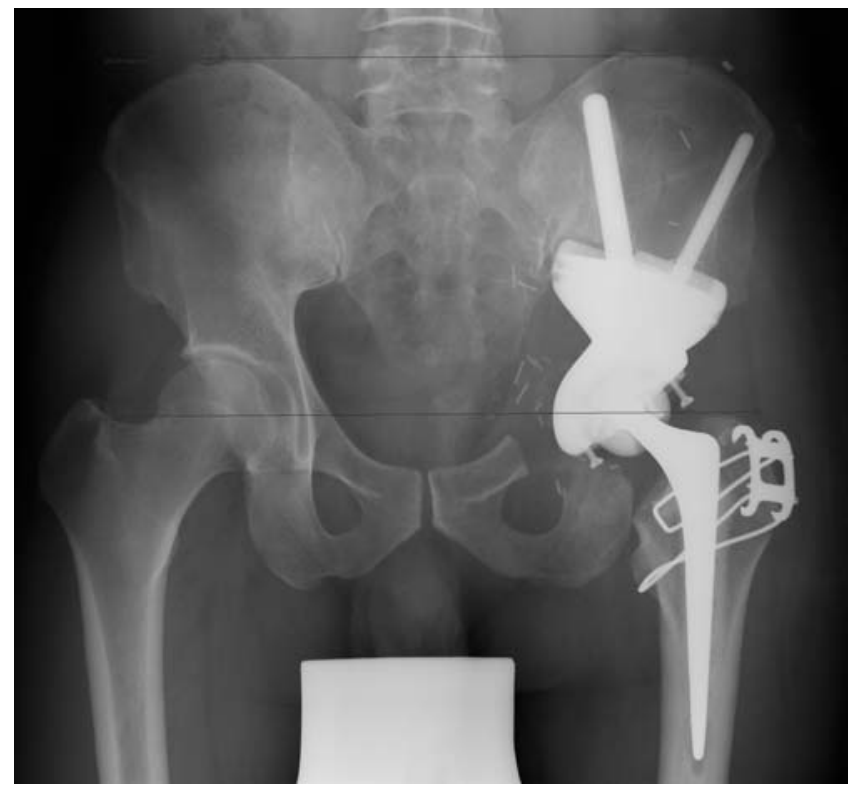

Fig. 4

Anteroposterior radiograph of pelvis taken two months after surgery showed satisfactory position of custom pelvic prosthesis in case 1 .

operatively by using a bone chisel or inserting two K-wires under navigational guidance. We found it easier and more precise to execute our pre-operative plan by identifying the location of pre-placed virtual screws under navigational guidance during surgery.

The navigation systems which are currently available have a number of drawbacks. We would, for example, like to have a 'navigation enabled' oscillating saw to improve the accuracy of resection.

On the software side, commercially available navigation systems do not allow direct transfer of electronic datasets from the CAD software used in the manufacture of custommade prostheses. The integration of CAD data into the navigation software would enable accurate resection and precise matching of the prosthesis to the resection. In cases 1 and 2 of our series, we had to transfer electronic measurements from the CAD dataset to the navigation software manually, so that the resection level and plane on which the CAD prosthesis was planned were represented identically in the navigation system.

Although additional operating time was needed for navigation, defining the resection plane on pre-operatively acquired images actually reduced the overall operating time, as we no longer had to establish the resection margins during surgery. We expect that the navigation time will lessen as surgeons become more familiar with the procedure.

The follow-up period of this study was short, and it remains to be seen whether computer-aided tumour resection and reconstruction achieves better oncological and functional results.

The use of a navigation system in musculoskeletal tumour resection has enabled the integration of pre-operative information about local anatomy and extent of the tumour, so that it is easier to identify resection margins precisely. It has enabled us to perform tumour resections that would otherwise have been difficult to achieve, and allowed accurate fitting of custom-made prostheses.

It would be helpful to have dedicated navigation software for musculoskeletal tumour surgery. This should allow the easy fusion of CT and MR images and the facility to inte- 
grate CAD data. The availability of tools to allow pre-operative planning and virtual resection on a console and to facilitate navigation along a resection plane would also be of great assistance.

We thank Mr Eric Wai-kin Ng and Mr Keith Kam-shing Lee (ACAOS-ITAV team, Department of Orthopaedics and Traumatology, Prince of Wales Hospital, Hong Kong) for the set-up of the navigation system and documentation of the operative procedures. We also appreciate the help of Professor Martin C. M. Wong and Mr Man-ho Lee (Department of Rapid Prototyping and Tooling Unit, Hong Kong Polytechnic University) for use of the computer-aided design software and fabrication of the plaster pelvic model.

No benefits in any form have been received or will be received from a commercial party related directly or indirectly to the subject of this article.

\section{References}

1. Grutzner PA, Suhm N. Computer aided long bone fracture treatment. Injury 2004;35(Suppl 1):57-64.
2. Gebhard F, Weidner A, Liener UC, Stockle U, Arand M. Navigation at the spine. Injury 2004;35(Suppl 1):35-45.

3. Laine T, Lund T, Ylikoski M, Lohikoshi J, Schlenzja D. Accuracy of pedicle screw insertion with and without computer assistance: a randomised controlled clinical study in 100 consecutive patients. European Spine J 2000;9:235-40.

4. Anderson KC, Buehler KC, Markel D. Computer assisted navigation in total knee arthroplasty: comparison with conventional methods. J Arthroplasty 2005;20(Suppl 3):132-8.

5. Wixson RL, MacDonald MA. Total hip arthroplasty through a minimal posterior approach using imageless computer-assisted hip navigation. J Arthroplasty 2005;20(Supp 3):51-6.

6. Enneking WF, Dunham W, Gebhardt MC, Malawer M, Pritchard D. A system for functional evaluation of reconstructive procedures after surgical treatment of tumors of the musculoskeletal system. Clin Orthop 1993;286:241-6.

7. Hufner T, Kfuri M Jr, Galanski M, et al. New indications for computer-assisted surgery: tumor resection in the pelvis. Clin Orthop 2004;426:219-25.

8. Krettek C, Geerling J, Bastian L, et al. Computer aided tumour resection in the pelvis. Injury 2004;35(Suppl 1):79-83. 